\title{
Lgr5+CD44+EpCAM + Strictly Defines Cancer Stem Cells in Human Colorectal Cancer
}

\author{
Zhengwei Leng ${ }^{\mathrm{a}} \quad$ Qinghua Xia $^{\mathrm{b}} \quad$ Jinhuang Chen ${ }^{\mathrm{b}}$ Yong Li ${ }^{\mathrm{b}} \quad$ Jiqian $\mathrm{Xu}^{\mathrm{b}}$ \\ Ende Zhao ${ }^{b}$ Hai Zheng ${ }^{b}$ Walden $\mathrm{Ai}^{\mathrm{c}}$ Jiangchuan Dong ${ }^{\mathrm{d}}$
}

aHepatobiliary, Pancreatic and Intestinal Diseases Research Institute, North Sichuan Medical College, Nanchong, 'bepartment of General Surgery, Union Hospital, Tongji Medical College, Huazhong University of Science and Technology, Wuhan, China, 'Department of Biology, Chemistry and Environmental Health science, Benedict College, Columbia, USA, dDepartment of Emergency, The Second Affiliated Hospital of Chongqing Medical University, Chongqing, China

\section{Key Words}

Lgr5 - CD44 - EpCAM - Colorectal cancer - Cancer stem cells - Biomarkers - Epithelialmesenchymal transition

\begin{abstract}
Background/Aims: Although EpCAM+CD44+ cells exhibit more stem-like properties than did EpCAM-CD44- cells, the specificity of EpCAM combined with CD44 in defining CSCs needs further improvement. Lgr5 is used as a biomarker to isolate cancer stem cells (CSCs) in colorectal cancer. However, it remains unclear whether Lgr5, along with EpCAM and CD44, can further identify and define CSCs in colorectal cancer. Methods: Lgr5+CD44+EpCAM+, Lgr5+CD44+EpCAM-, Lgr5+CD44-EpCAM+, Lgr5-CD44+EpCAM+, and Lgr5-CD44-EpCAMcells were separately isolated using fluorescence-activated cell sorting (FACS). Colony formation, self-renewal, differentiation, and tumorigenic properties of these cells were investigated through in vitro experiments and in vivo tumor xenograft models. The expression of stemness genes and CSC- and epithelial-mesenchymal transition (EMT)-related genes, such as KLF4, Oct4, Sox2, Nanog, CD133, CD44, CD166, ALDH1, Lgr5, E-cadherin, ZO-1, Vimentin, Snail, Slug, and Twist, was examined using real-time PCR. Results: Lgr5-positive subpopulations exhibited higher capacities for colony formation, self-renewal, differentiation, and tumorigenicity as well as higher expression of stemness genes and mesenchymal genes and lower expression of epithelial genes than did Lgr5-negative subpopulations. Conclusion: Our data revealed that tumorigenic cells were highly restricted to Lgr5-positive subpopulations. Most importantly, Lgr5+CD44+EpCAM+ cells exhibited more pronounced CSC-like traits than did any other subpopulation, indicating that Lgr5 combined with CD44 and EpCAM can further improve the stem-like traits of CSCs in colorectal cancer.




\section{Introduction}

Colorectal cancer is a genetic disease characterized by uncontrolled cell growth and the ability to invade other parts of the body by forming metastases [1]. Emerging evidence has shown that a subpopulation of cells, cancer stem cells (CSCs) or tumor-initiating cells, displays stem cell characteristics that influence tumorigenesis [2]. These cells possess specific properties, including self-renewal, differentiation potential, resistance to chemotherapy and high tumorigenicity. Further evidence has suggested an association between CSC phenotype and the progression of the epithelial-to-mesenchymal transition (EMT) $[3,4]$, whereby epithelial cells lose their epithelial features and acquire mesenchymal features. More importantly, cancer cells can become more aggressive through the acquisition of mesenchymal properties and can thus promote tumor metastasis and progression [5-7].

CSCs have been prospectively identified in colorectal cancer by their expression of specific surface markers, such as leucine-rich-repeat-containing G-protein-coupled receptor 5 (Lgr5), CD133, CD44, CD166, EpCAM (epithelial cell adhesion molecule), CD24, CD29, and aldehyde dehydrogenase (ALDH), among others [2, 8-10]. More recently, it was reported that Lgr5 showed promise as a biomarker for colorectal CSCs $[9,11,12]$. Moreover, increasing evidence has shown that Lgr5 is involved in the Wnt/ $\beta$-catenin pathway, which plays a key role in maintaining CSC self-renewal and tumorigenic ability [13-15]. Therefore, Lgr5 seems to be a valuable biomarker for identifying and isolating human colorectal CSCs during cancer progression. However, it remains unclear whether Lgr5, along with other CSC markers, can further enhance tumor-initiation capacity and define CSCs in colorectal cancer.

EpCAM is expressed in many human cancers of epithelial origin. A recent study indicated that EpCAM is expressed in colorectal CSCs $[16,17]$. Furthermore, Liu et al. demonstrated that EpCAM ${ }^{\text {high }} / \mathrm{CD} 44+$ cells were significantly correlated with the invasion and metastasis of colorectal cancer [18]. CD44 is a widely expressed protein with a role in cell-to-cell and cell-to-matrix interactions. CD44 overexpression increases tumor growth and anti-apoptotic properties. A recent study showed that CD133+CD44+ cells isolated from human colorectal cancer cell line exhibited CSC properties [2].

It is generally considered that the identification and characterization of colorectal CSCs may lead to revolutionary findings in targeted therapeutic strategies [19-21]. However, a long-standing problem has been the paucity of specific markers with which to identify and isolate colorectal CSCs and to investigate their involvement in cancer progression [22]. To determine more specific CSC markers, in this study, we first examined whether colorectal CSCs could be further identified and defined using Lgr5 combined with CD44 and EpCAM.

\section{Materials and Methods}

\section{Ethics statement}

This study was conducted in strict accordance with the protocol approved by the Committee on the Ethics of Animal Experiments of the University of Huazhong University of Science and Technology (Permit Number: S255). All surgery was performed under a mixture of ketamine and chlorpromazine anesthesia, and all efforts were made to minimize suffering [8].

Cell lines and culture conditions

The human colorectal cancer cell line DLD-1 (CCL-221) was obtained from the American Type Culture Collection (ATCC, Manassas, VA, USA). Cells were cultured in RPMI 1640 medium (HyClone, GE Healthcare Life Sciences, Logan, Utah, USA) supplemented with 10\% fetal bovine serum (FBS, Gibco, Thermo Fisher Scientific, Inc., Waltham, MA, USA) and were maintained in a humidified incubator at $37^{\circ} \mathrm{C}$ and $5 \% \mathrm{CO}_{2}$ [8].

Flow cytometric analysis and cell sorting

Cells were trypsinized with Accutase (Innovative Cell Technologies, San Diego, CA, USA) and resuspended in staining buffer $\left(1 \times 10^{6}\right.$ cells in $\left.80 \mu \mathrm{l}\right)$. Cells were then treated with $20 \mu \mathrm{l}$ of FcR Blocking 


\section{Cellular Physiology Cell Physiol Biochem 2018;46:860-872

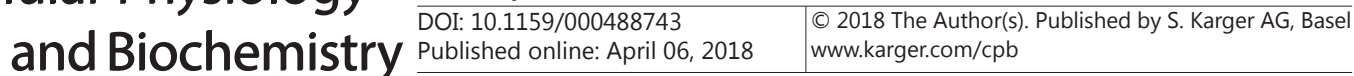

Leng et al.: Lgr5+CD44+EpCAM+ Defines CSCs

Reagent at room temperature for $15 \mathrm{~min}$, followed by incubation with antibodies (human anti-CD44-APC and human anti-EpCAM-Alexa Fluor 488, eBioscience, Thermo Fisher Scientific, MA, USA; and human antiLgr5-PE, BD, San Jose, CA, USA) at $4^{\circ} \mathrm{C}$ for $30 \mathrm{~min}$. Then, 7AAD staining was performed to exclude dead cells. After staining, cells were subjected to flow cytometry analysis using BD FACSVerse (BD, USA). For sorting, cells were collected using a BD FACSAria Fusion flow cytometer (BD, USA) and then cultured in serum-free DMEM/F12 medium containing $20 \mathrm{ng} / \mathrm{ml}$ epidermal growth factor (EGF, PeproTech, Inc., Rocky Hill, NJ, USA), $10 \mathrm{ng} / \mathrm{ml}$ basic fibroblast growth factor (bFGF, PeproTech, USA), 5 gg/ml insulin (Sigma, Merck kGaA, Darmstadt, Germany), 0.4\% bovine serum albumin (BSA, Amresco, Solon, OH, USA), 100 U/ml Pen/Strep (HyClone, USA), and 2\% B27 (Invitrogen, Thermo Fisher Scientific, MA, USA) for 2 days before transfection.

\section{Sphere-forming assay}

Single cells (500 cells per well) were plated in 6-well plates and cultured in serum-free DMEM/F12 medium (as detailed above) for 7 days. At the end of the experiment, the number of spheres per well was counted.

\section{Limiting dilution assay}

The LDA and tumor sphere-forming assays were performed as previously described, with some modifications [23]. In brief, limiting dilutions of tumor cells were plated (4 to 0.5 cells per well) in $0.2 \mathrm{ml}$ of serum-free DMEM/F12 medium as described for the sphere-forming assay and were cultured for 7 days. At the end of the experiment, the number of spheres per well was counted. The fraction of negative wells compared with the cell dilution was graphed and fitted with a linear regression model to estimate CSC frequency. Following the assumption that a single stem cell gives rise to one sphere [24], the proportion of negative wells was defined by the zero point $\left(\mathrm{F}_{0}\right)$ of the Poisson distribution: $\mathrm{F}_{0}=e^{-x}$, where $x$ is the mean number of cells per well. One CSC (one sphere) was expected at a dilution of 0.37 (when $x=1, \mathrm{~F}_{0}=e^{-1}=0.37$ ).

\section{Colony formation assay}

The colony formation assay was performed as previously described [8]. Briefly, single cells (500 cells per well) were plated in 6-well plates and cultured for 2 weeks. After crystal violet staining, the cells were photographed, and the in vitro tumorigenic efficiency was analyzed.

\section{Immunofluorescence staining and laser confocal microscopy}

Lgr5+CD44+EpCAM+ cells were sorted and smeared before being fixed with $2 \%$ paraformaldehyde at $37^{\circ} \mathrm{C}$ for $15 \mathrm{~min}$. The fixed cells were then permeabilized with $0.1 \%$ Triton X-100 at room temperature for $15 \mathrm{~min}$, followed by incubation at $4^{\circ} \mathrm{C}$ for $10 \mathrm{~h}$ with primary antibodies against Vimentin $(1: 200$, Cell Signaling Technology, MA, USA) and Lgr5 (1:200, Santa Cruz Biotechnology, CA, USA). The cells were then incubated with Alexa Fluor 488- (1:200, Cell Signaling Technology, USA) or DyLight 649-conjugated secondary antibodies $\left(1: 200\right.$, Multi Sciences, China) at $37^{\circ} \mathrm{C}$ for $30 \mathrm{~min}$. These slides were then mounted with Prolong Gold with 4,6-diamidino-2-phenylindole (DAPI, Invitrogen, USA). Fluorescent images were obtained using an FV1200 laser confocal microscope (Olympus, Tokyo, Japan).

\section{Real-time migration and invasion assay}

The migration and invasion rates of each population were assessed using the xCELLigence system (RTCA DP Station, Roche, IN, USA) as previously described [25, 26]. This assay provides a real-time measurement of each population's migration and invasion by extrapolating the number of cells passing through a porous membrane from the electrical impedance data. Briefly, $150 \mu \mathrm{l}$ of RPMI 1640 complete medium was added to the lower chamber of a cellular migration and invasion plate (Roche). The upper chamber was coated with (invasion) or without (migration) $10 \mu \mathrm{l}$ of Matrigel (Sigma) and incubated for $15 \mathrm{~h}$. The upper chamber was then loaded with $50 \mu \mathrm{l}$ of serum-free RPMI 1640 medium and fitted onto the lower chamber. After $30 \mathrm{~min}$ of equilibration, $2 \times 10^{4}$ cells from each subpopulation were plated onto each well of the upper chamber. The plates were subsequently placed on the RTCA analyzer under humidified conditions at $37^{\circ} \mathrm{C}$ and $5 \% \mathrm{CO}_{2}$. The cellular migration and invasion of each subpopulation was recorded every $20 \mathrm{~min}$.

\section{Cell proliferation assay}

Proliferation capacity was analyzed using a CCK-8 assay kit (DOJINDO, Tokyo, Japan) according to the manufacturer's instructions. Briefly, $5 \times 10^{4}$ cells per well from each subpopulation were seeded into 96- 


\section{Cellular Physiology Cell Physiol Biochem 2018;46:860-872 \begin{tabular}{ll|l} 
and Biochemistry Published online: April 06, 2018 & $\begin{array}{l}\text { (c) } 2018 \text { The Author(s). Published by S. Karger AG, Basel } \\
\text { www.karger.com/cpb }\end{array}$
\end{tabular} \\ Leng et al.: Lgr5+CD44+EpCAM+ Defines CSCS}

well plates containing complete medium and cultured for $72 \mathrm{~h}$. The absorption (OD value) at $450 \mathrm{~nm}$ was measured for each well using a plate reader and then graphed [27].

\section{Soft agar assay}

The soft agar assay was performed as previously described [8]. Briefly, each well of a 6-well plate was loaded with $2 \mathrm{ml}$ of $0.5 \%$ agar $(\mathrm{w} / \mathrm{v}$ ) in RPMI 1640 supplemented with $10 \%$ FBS as a base. After polymerization of the base agar, $1 \times 10^{3}$ cells mixed in $2 \mathrm{ml}$ of $0.375 \%$ agar (w/v) in RPMI 1640 supplemented with 10\% FBS were added. Cultures were maintained in a humidified incubator at $37^{\circ} \mathrm{C}$ and $5 \% \mathrm{CO}_{2}$ for 2 weeks before colonies were counted and photographed.

\section{RNA isolation and $q R T-P C R$}

Total mRNA was extracted from cells and then reverse-transcribed to cDNA with PrimeScript RT Master Mix (Roche) according to the manufacturer's instructions. qRT-PCR was performed to quantify the expression of stemness genes and EMT genes using LightCycler FastStart DNA Master ${ }^{\text {PLUS }}$ SYBR Green I (Roche) according to the manufacturer's protocol on a Roche LightCycler 96 (Roche). Glyceraldehyde-3phosphate-dehydrogenase (GAPDH) was used as an internal control for the normalization of these genes. PCR amplification was conducted under the following conditions: 1 cycle of pre-incubation at $95^{\circ} \mathrm{C}$ for 10 min, followed by 40 cycles of denaturation for $10 \mathrm{sec}$ at $95^{\circ} \mathrm{C}$, annealing for $10 \mathrm{sec}$ at $60^{\circ} \mathrm{C}$, and extension for $10 \mathrm{sec}$ at $72^{\circ} \mathrm{C}$. Primer sequences and specifications are summarized in Table 1 . For each sample, duplicate reactions were performed, and the average mRNA level of each gene was determined using the 2- $\Delta \Delta \mathrm{Ct}$ method with the Lgr5-CD44-EpCAM- subpopulation as a control group.

\section{In vivo tumor cell xenograft model}

All animal experiments were approved by the Ethics Committee of Huazhong University of Science and Technology (\#S255; Wuhan, China). A total of $5 \times 10^{5}$ cells in $100 \mathrm{ml}$ of PBS were subcutaneously transplanted into 4-week-old female Balb/c nu/nu mice ( $n=5$ mice per group) (Beijing HFK Bioscience Co., Ltd, China). Mice were housed in a specific-pathogen-free, environmentally controlled facility [28]. Mice were sacrificed with an intraperitoneal injection of pentobarbital sodium (100 mg/kg, Sigma), and grafts were removed when the tumors reached a length of $2.0 \mathrm{~cm}$ or 60 days after injection, whichever occurred first [8]. Harvested tumors were prepared for histopathologic analysis. Tumor volume was calculated using the following formula: tumor volume=length $\times$ width $^{2} \times 0.5$.

\section{Statistical analysis}

Statistical analyses were performed using SPSS software, version 19.0. All data with a normal distribution are presented as the means \pm s.d., comparisons among groups were analyzed using one-way ANOVA, and pairwise comparisons were performed using a paired-samples $t$-test. Data with a value of $P<0.05$ were considered statistically significant.

\section{Results}

Lgr5 strictly defines stemness in EpCAM+CD44+ and EpCAM-CD44-subpopulations

Some researchers demonstrated that EpCAM and CD44 expression were significantly correlated with the invasion and metastasis of colorectal cancer [18]. Although EpCAM and CD44 are thought to act as a marker of colorectal CSCs, the stem-like properties of
Table 1. Primers used for qRT-PCR amplification of each mRNA

\begin{tabular}{lr}
\hline Primer & sequence \\
\hline GAPDH-F & 5'-GGGGAGCCAAAAGGGTCATCATCT-3' \\
GAPDH-R & 5'-GACGCCTGCTTCACCACCTTCTTG-3' \\
KLF4-F & 5'-CGAACCCACACAGGTGAGAA-3' \\
KLF4-R & 5'-TACGGTAGTGCCTGGTCAGTTC-3' \\
Oct4-F & 5'-CTTGCTGCAGAAGTGGGTGGAGGAA-3' \\
Oct4-R & 5'-CTGCAGTGTGGGTTTCGGGCA-3' \\
Sox2-F & 5'-CAAGATGCACAACTCGGAGA-3' \\
Sox2-R & 5'-CATGAGCGTCTTGGTTTTCC-3' \\
Nanog-F & 5'-CAGAAGGCCTCAGCACCTACCTACCCCAGCC-3' \\
Nanog-R & 5'-TCTCTGCAGTCCTGCATGCAGTTCCAGCCAAA-3' \\
CD133-F & 5'-ACCGACTGAGACCCAACATC-3' \\
CD133-R & 5'-GACCGCAGGCTAGTTTTCAC-3' \\
CD44-F & 5'-CAACACAAATGGCTGGTACG-3' \\
CD44-R & 5'-GTGTGGTTGAAATGGTGCTG-3' \\
CD166-F & 5'-CGCAATGCAACAGGAGACTA-3' \\
CD166-R & 5'-CCACAGTTGCATTCCTGCTA-3' \\
ALDH1-F & 5'-TGTTAGCTGATGCCGACTTG-3' \\
ALDH1-R & 5'-CTTCTTAGCCCGCTCAACAC-3' \\
Lgr5-F & 5'-CTCTTCCTCAAACCGTCTGC-3' \\
Lgr5-R & 5'-GATCGGAGGCTAAGCAACTG-3' \\
E-cad-F & 5'-GCCCTGCCAATCCCGATGAAA-3' \\
E-cad-R & 5'- GGGGTCAGTATCAGCCGCT-3' \\
ZO-1-F & 5'- TGAGGCAGCTCACATAATGC-3' \\
ZO-1-R & 5'-GGGAGTTGGGGTTCATAGGT-3' \\
Vim-F & 5'-GCTTCAGAGAGAGGAAGCCGAAAA-3' \\
Vim-R & 5'-CCGTGAGGTCAGGCTTGGAAA-3' \\
Snail-F & 5'-CCAGACCCACTCAGATGTCAAGAA-3' \\
Snail-R & 5'-GGCAGAGGACACAGAACCAGAAAA-3' \\
Slug-F & 5'-CCCAATGGCCTCTCTCCTCTTT-3' \\
Slug-R & 5'-CATCGCAGTGCAGCTGCTTATGTTT-3' \\
Twist-F & 5'-CTAGAGACTCTGGAGCTGGATAACTAAAAA-3' \\
Twist-R & 5'-CGACCTCTTGAGAATGCATGAAAAA-3' \\
\hline &
\end{tabular}


EpCAM+CD44+ cells remain unclear.Therefore, wefirstsorted $\mathrm{EpCAM+CD44+}$ and EpCAMCD44- (Fig. 1A) cells from DLD1 cells and demonstrated that EpCAM+CD44+ cells exhibit more stem-like properties than did EpCAM-CD44- cells (Fig. 1B). However, a portion of EpCAM+CD44+ cells did not generated spheres, while a portion of EpCAM-CD44- cells generated spheres, indicating that the specificity of EpCAM combined with CD44 is not sufficient to define CSCs and needs to be further improved (Fig. 1B).

We then asked whether Lgr5 could further define CSCs in EpCAM+CD44+ and EpCAMCD44- subpopulations. Lgr5+ and Lgr5- cells were sorted from both EpCAM+CD44+ and EpCAM-CD44- subpopulations. As shown in Fig. 1C, the Lgr5+ cells sorted from the two subpopulations generated spheres and colonies, while Lgr5- cells rarely generated either spheres or colonies. These data indicated that Lgr5 strictly defines stemness in both EpCAM+CD44+ and EpCAMCD44- subpopulations. However, the Lgr5+EpCAM+CD44+ subpopulation generated more spheres and colonies than the Lgr5+EpCAM-CD44- subpopulation.

To further study whether Lgr5 combined with CD44 and EpCAM could improve the specificity of defining CSCs, the expression of CSC markers (including Lgr5, CD44, and EpCAM) was analyzed in human colorectal cancer cells and used for sorting by flow cytometry $[8,9,18]$. For convenience, we designated Lgr5+CD44+EpCAM+, Lgr5+CD44+EpCAM-, Lgr5+CD44-EpCAM+, Lgr5-CD44+EpCAM+, and Lgr5-CD44EpCAM- cells as subpopulations 1-5 (S1-S5), respectively. The percentages of each subpopulation are summarized in Table 2. Sorted cells (S1-S5) were used for further study.

\section{Expression of stemness genes and CSC genes in each subpopulation}

To understand differences in the expression of stemness genes and EMT genes in each subpopulation, quantitative real-time PCR (qRT-PCR) was performed to investigate mRNA levels in these cells. The results in Fig. 2 indicate that both stemness genes, such as Oct4, Sox2, Nanog, and KLF4, and CSC genes, such as CD133, CD44, CD166, ALDH1, and Lgr5, were more highly expressed in Lgr5-positive subpopulations. Moreover, stemness genes were extremely overexpressed in Lgr5+CD44+EpCAM+ (S1) compared with their expression in the other subpopulations. 
Self-renewal properties and frequencies of CSCs in each subpopulation

The defining feature of CSCs is self-renewal, which is responsible for cancer recurrence and metastasis $[16,29]$. To examine the selfrenewal properties of each subpopulation, we performed sphere-forming assays. Our data demonstrated that Lgr5positive cells (S1, S2, and S3) generated significantly more multipotent spheres than did Lgr5-negative cells (S4 and S5). More importantly, the S1 subpopulation generated the maximum number of spheres, indicating a selfrenewal advantage in Lgr5+CD44+EpCAM+ cells (Fig. 3A).

Since a sphere is thought to represent all the progeny from a single stem cell, sphere formation reflects the stem cell population [30]. Thus, CSC frequency can be estimated through a limiting dilution assay [24]. Our data showed that the median CSC frequencies of the $S 1(10 / 121)$, S2 (10/133), and S3 (10/137) subpopulations were much higher than those of the S4 $(10 / 238)$ and S5 $(10 / 250)$ subpopulations (Fig. 3B).

Fig. 3. Characterization of self-renewal and stem-like properties. (A) When cultured in serum-free medium, Lgr5-negative cells (S4 and S5) formed smaller spheres at a significantly lower frequency than did Lgr5-positive cells (S1, S2 and S3). (B) The CSC median frequencies of the S1 (10/121), S2 (10/133), and S3 (10/137) subpopulations were higher than those of the S4 $(10 / 238)$ and $5(10 / 250)$ subpopulations, any other group.
Fig. 2. mRNA expression of stemness genes and epithelialto-mesenchymal transition (EMT) genes. (A) Stemness genes, such as Oct4, Sox2, Nanog and KLF4, were expressed more highly in Lgr5positive cells (S1, S2 and S3) than in Lgr5negative cells (S4 and S5), as assessed by qRT-PCR. CSC genes, such as CD133, CD44,
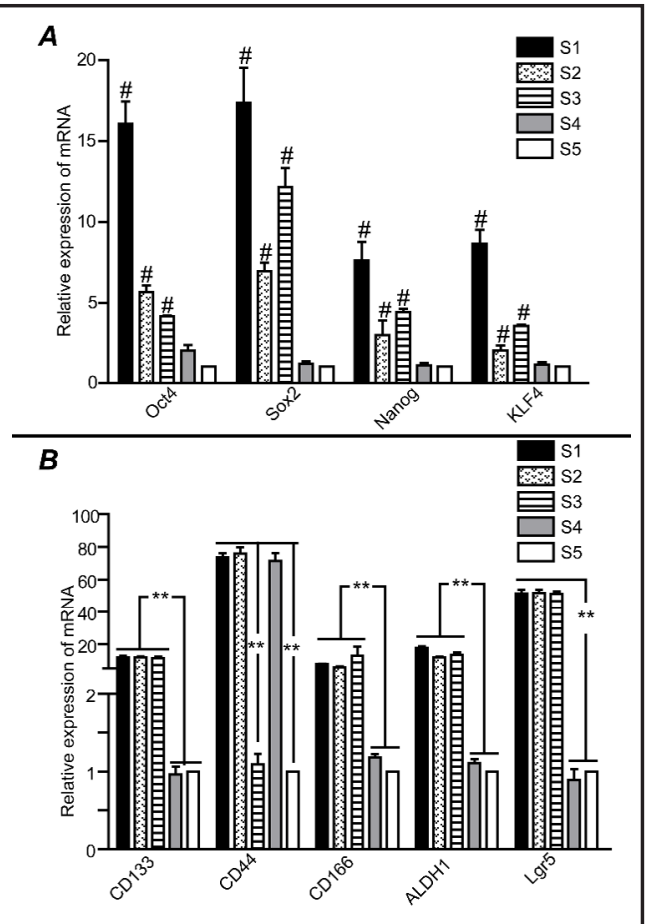
CD166, ALDH1, and Lgr5, were expressed more highly in Lgr5-positive cells than in Lgr5-negative cells, except for CD44 expression in the S3 subpopulation. \# $\mathrm{P}<0.05$ compared to any other group. ${ }^{* *} \mathrm{P}<0.05$. The S5 subpopulation was used as a control group.

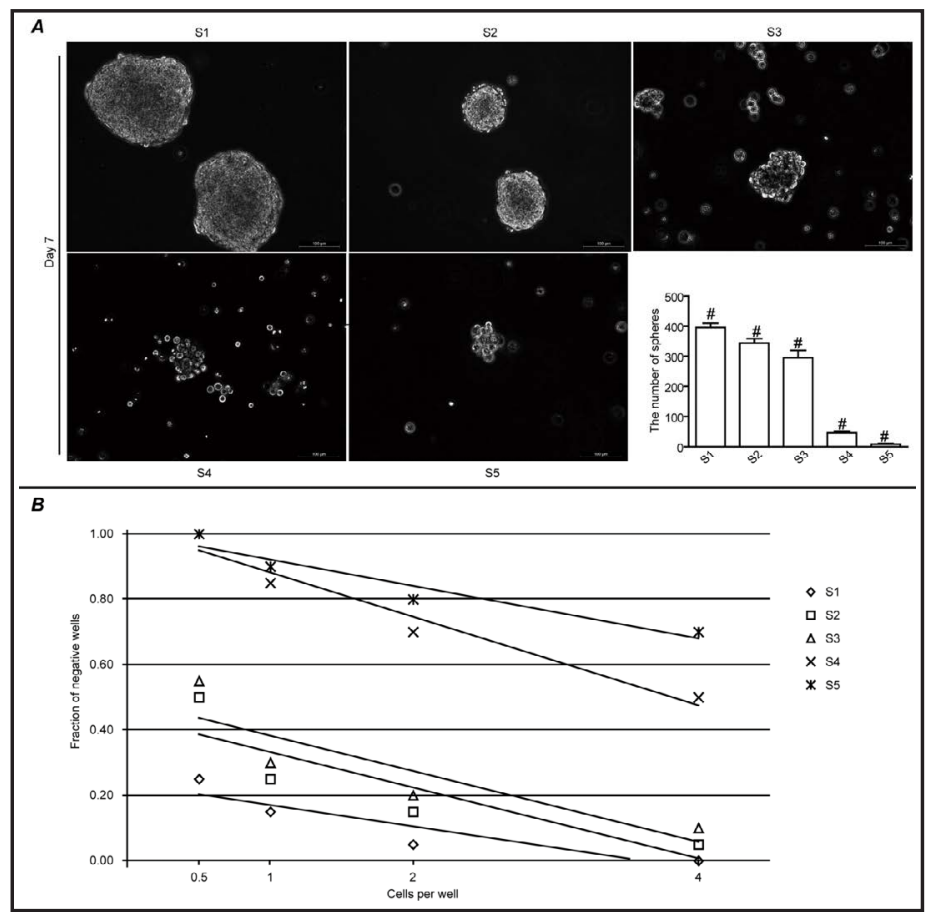




\section{Cellular Physiology Cell Physiol Biochem 2018;46:860-872 \begin{tabular}{l|l} 
DOI: 10.1159/000488743 \\
and Biochemistry Published online: April 06, 2018 & $\begin{array}{l}\text { O } 2018 \text { The Author(s). Published by S. Karger AG, Basel } \\
\text { www.karger.com/cpb }\end{array}$ \\
\cline { 2 - 3 }
\end{tabular} Leng et al.: Lgr5+CD44+EpCAM+ Defines CSCs}

Fig. 4. Characterization of colony formation and proliferation properties. (A) Soft agar assays showed that Lgr5positive cells (S1, S2 and S3) generated more colonies than did Lgr5-negative cells (S4 and S5). (B) Colony formation assays showed that Lgr5-positive cells (S1, S2 and S3) generated more colonies than did Lgr5negative cells (S4 and S5). (C) The CCK-8 assay indicated that Lgr5-positive cells exhibited a significantly higher proliferative capacity than did Lgr5negative cells. $\# \mathrm{P}<0.05$ compared to any other group.

Colony formation and proliferation properties in each subpopulation

We then investigated the malignant profile of each subpopulation, including its ability to form colonies and proliferate [8, 31]. We found that the S1, S2 and S3 subpopulations had a significantly higher colony formation capacity than did the S4 and S5 subpopulations, as assessed by the soft agar assay and colony formation assay (Fig. 4A-4B). S1 cells generated the maximum number of colonies, indicating an advantage of Lgr5+CD44+EpCAM+ cells in colony formation and proliferation.
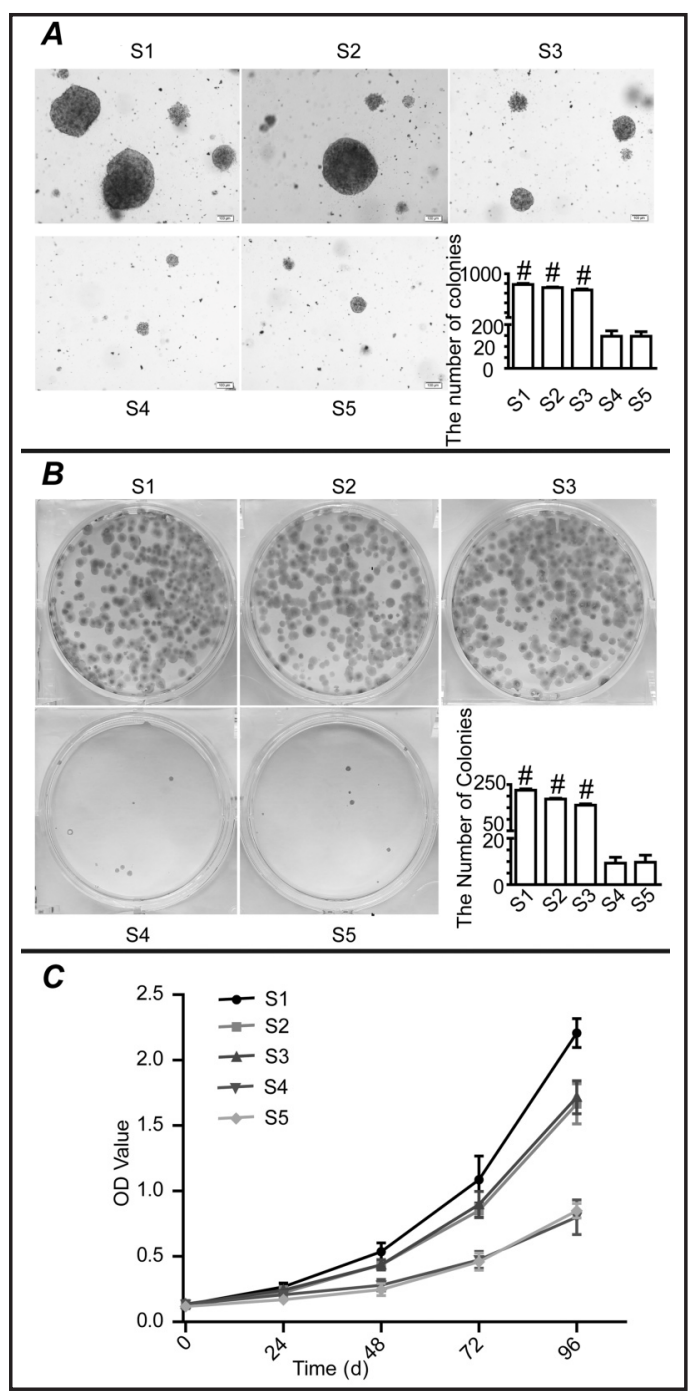

Fig. 5. Characterization of epithelial-mesenchymal transition profiles. (A) Mesenchymal genes, such as Vimentin, Snail, Slug, and Twist, were more highly expressed in Lgr5-positive cells ( $\mathrm{S} 1, \mathrm{~S} 2$ and S3) than in Lgr5-negative cells (S4 and S5), while the epithelial genes E-cadherin and Z0-1 were more highly expressed in Lgr5-negative cells, as assessed by qRT-PCR. (B) Immunofluorescence staining and laser confocal microscopy demonstrated the co-expression of Lgr5 and Vimentin in S1 cells. (C) Lgr5positive cells exhibited a higher capacity for migration and invasion

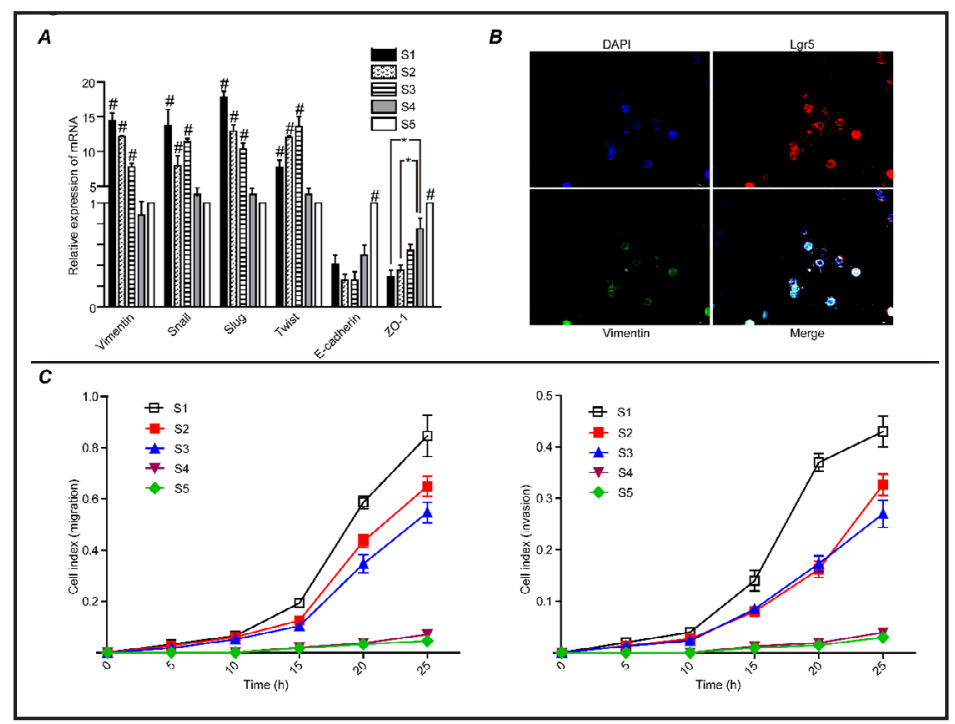
than did Lgr5-negative cells, as assessed by real-time migration and invasion assays. Bar, $30 \mu \mathrm{m}$. \# $\mathrm{P}<0.05$ compared to any other group. The S5 subpopulation was used as a control group. 
We then evaluated the proliferation capacity of each subpopulation using the CCK8 assay [8]. We cultured $2 \times 10^{3}$ cells from each subpopulation in 96-well plates to compare cell proliferation and repeated this assay 10 times. The results in Fig. 4C indicated that Lgr5-positive cells (S1, S2, and S3) exhibited a significantly higher proliferation capacity than did Lgr5-negative cells (S4 and S5). Additionally, Lgr5+CD44+EpCAM+ (S1) cells could proliferate more rapidly than any other subpopulation.

Mesenchymal characteristics in each subpopulation

EMT has been reported as a mechanism by which can-

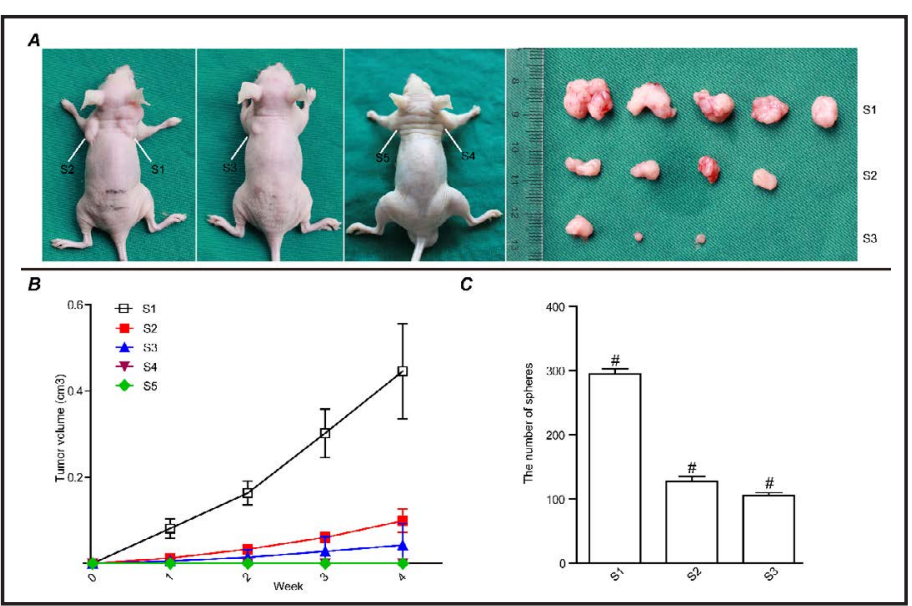

Fig. 6. Characterization of tumor-initiating capacity. (A-B) On day 60 , mice transplanted with S1 cells exhibited earlier tumor formation and significantly larger tumors than mice transplanted with S2 or S3 cells; no tumor growth was observed after the injection of S4 or S5 cells. (C) S1 cells formed larger spheres at a significantly higher frequency than did S2 or S3 cells. \# $<0.05$ compared to any other group. cer cells acquire invasive and stem-like characteristics [3]. Some researchers have also demonstrated a partial overlap between mesenchymal characteristics and CSC characteristics $[3,4]$. Thus, we investigated mesenchymal characteristics, such as EMT-related gene expression, migration and invasion, in each subpopulation. As shown in Fig. 5A, mesenchymal genes, such as Vimentin, Snail, Slug, and Twist, were more highly expressed in Lgr5-positive cells (S1, S2 and S3) than in Lgr5-negative cells (S4 and S5), while the epithelial genes E-cadherin and ZO-1 were more highly expressed in Lgr5-negative cells. Moreover, the co-expression of Lgr5 and Vimentin in S1 cells was confirmed by immunofluorescence (Fig. 5B). Finally, we demonstrated that Lgr5-positive cells (S1, S2 and S3) exhibited a higher capacity for migration and invasion than did Lgr5-negative cells (S4 and S5), as assessed by real-time migration and invasion assays, respectively. More importantly, the $\mathrm{S} 1$ subpopulation could migrate and invade more rapidly than other subpopulations (Fig. 5C).

\section{Tumor-initiating capacity of cell subpopulations expressing CSC markers}

Finally, the tumor formation capacity of the putative CSC subpopulations was evaluated in immunodeficient mice [32]. Titrated numbers of triple-positive (S1) cells and their counterparts were subcutaneously injected into C57BL/ 6 nude mice. The injected S2 and S3 cells displayed significantly delayed tumor growth compared with that of the triple-positive cells. Most importantly, no tumor growth was observed after the injection of $1 \times 10^{7} \mathrm{~S} 4$ and S5 cells (Fig. 6A-6B). To further assess the effects of CSC subpopulations in tumor xenografts, single cells derived from xenograft tumors were replated in sphere culturing conditions. As expected, the sphere-forming capacity of Lgr5+CD44+EpCAM+ cells was sharply increased compared with that of the S2 and S3 cells, indicating a higher capacity for self-renewal (Fig. 6C). Collectively, these results indicated that Lgr5-positive cells exhibited higher tumorigenic potential than did Lgr5-negative cells in vivo and that the Lgr5+CD44+EpCAM+ subpopulation exhibited a higher tumor-initiating capacity than did the other subpopulations.

\section{Discussion}

The metastasis and recurrence associated with human colorectal cancer plays a key role in cancer-related mortality $[1,33]$. Increasing evidence suggests that only a small 
subpopulation of cancer cells can regenerate [22]. These cells, termed CSCs, are thought to cause the initiation, therapeutic resistance, metastasis, and recurrence of cancer [2, 34, 35]. Accordingly, it is crucial to identify markers specific to CSCs to facilitate the development of targeted cancer therapies $[5,16]$. The objectives of this study were to evaluate the expression of surface biomarkers (including Lgr5, CD44, and EpCAM) in human colorectal CSCs and to investigate their correlation with functional stem-like characteristics. In recent years, studies have revealed that Lgr 5 is overexpressed in various tumor types, including colorectal cancer, hepatocellular carcinoma, and esophageal adenocarcinoma $[9,11,13,36]$. Studies in mice have demonstrated that Lgr5 might be another marker of both normal colon stem cells and colon CSCs [37]. Indeed, Lgr5 is a member of the Wnt/ $\beta$-catenin pathway, which plays a key role in maintaining CSC self-renewal and tumorigenic ability $[9,13,38,39]$. Kemper et al. isolated a distinct subpopulation of EpCAM+Lgr5+ cells from primary human colorectal cancer cells and confirmed that Lgr5 overexpression induced the malignant cancer profile [9]. EpCAM was reported to be overexpressed in human breast and colon CSCs [16]. Moreover, Yamashita et al. found that EpCAM expression was regulated by the Wnt/ $\beta$-catenin pathway and that EpCAM+ hepatocellular carcinoma cells were tumor-initiating cells with stem-like features [40]. Thus, EpCAM may act downstream of the Wnt/ $\beta$-catenin pathway to maintain stemness and could serve as a useful marker for colorectal CSCs. Recently, Liu et al. reported that the presence of EpCAM+CD44+ cells was significantly correlated with the depth of invasion, clinical stage, degree of differentiation, and metastatic status in colorectal cancer [18]. In addition, double-positive cells were detected in the corresponding liver metastases [18]. Previous studies have confirmed that EpCAM+CD44+ colorectal cancer cells have stemlike properties, leading to the tumorigenesis, migration, invasion and metastasis of human colorectal cancer $[16,41,42]$. These data suggest that EpCAM+CD44+ may be used as a reliable marker of colorectal CSCs [16]. More importantly, scientists have demonstrated that the combined and sequential use of CSC markers led to higher purified CSC subpopulations than any single biomarker $[43,44]$. To date, no study has investigated the characteristics of colorectal CSCs expressing Lgr5 combined with CD44 and EpCAM. In this study, we performed a systematic evaluation to better characterize and determine the utility of Lgr5 along with CD44 and EpCAM in identifying CSCs from the DLD-1 human colorectal cancerderived cell line.

The critical feature of CSCs is self-renewal, which is responsible for cancer drug resistance, metastasis, and recurrence $[19,20,45]$. We found that Lgr5-positive subpopulations generated significantly more multipotent spheres than did Lgr5-negative subpopulations, indicating a remarkable self-renewal advantage of Lgr5-positive cells. Moreover, the Lgr5+CD44+EpCAM+ subpopulation displayed a higher self-renewal capacity than did any other subpopulations (Fig. 3A). We also performed a traditional and reliable limiting dilution assay (LDA) to determine the fraction of CSCs in each subpopulation. The LDA results revealed the frequency of sphere formation, providing useful information for interpreting the composition of the mixed population of CSCs $[23,46]$. Our current data showed that Lgr5-positive subpopulations possessed a higher percentage of CSCs than did Lgr5-negative subpopulations. The CSC median frequencies of S1 (10/121), S2 (10/133), and S3 (10/137) were higher than those of the S4 (10/238) and S5 (10/250) subpopulations. The CSC fraction was dramatically increased in the Lgr5+CD44+EpCAM+ subpopulation compared with that of the other subpopulations (Fig. 3B).

Stemness genes, such as Oct4, Sox2, c-Myc, and KLF4, have been recognized as "pluripotency factors" that play critical roles in maintaining the self-renewal capacity of stem cells [47]. We demonstrated that the expression of stemness genes was higher in Lgr5positive cells and markedly increased in Lgr5+CD44+EpCAM+ cells compared with that in other subpopulations, which is consistent with the data from the LDA and the sphere-forming assay (Fig. 2A). Previous studies have demonstrated correlations between the maintenance of human CSC stemness and the expression of multipotency genes [48-50]. Saiki et al. showed that Sox2 expression was highly associated with lymph node metastasis and Dukes stage in 
human colorectal cancer [50]. Another study revealed correlations between the expression of Oct4, Sox2, and CD133 and poor prognosis of colorectal cancer patients [49].

The EMT has been postulated as a mechanism by which cancer cells acquire the invasive and stem-like traits necessary for distant metastasis [3]. The EMT process is characterized by decreases in epithelial genes, such as ZO-1 and E-cadherin, combined with the acquisition of mesenchymal markers, such as Vimentin, Snail and Slug [6, 8]. More importantly, the EMT is correlated with increases in malignant cancer phenotypes, such as migration, invasion, and metastasis [51]. Decreased expression of mesenchymal markers or the re-expression of E-cadherin was shown to reduce the cancer invasion phenotype [31, 51], indicating that these genes play a critical role in controlling the malignant profiles of cancer cells. Some researchers have indicated that CSCs might be generated through processes that are related to activation of the EMT $[3,4]$. Thus, CSC biology and the EMT are thought to be mechanistically correlated and key components of cancer migration, invasion, and metastasis [5, 52-54]. Accordingly, in-depth research into the crosstalk between cancer stemness and the EMT is essential for a better understanding of tumor progression. Our findings also demonstrated that Lgr5-positive cells displayed higher expression of Vimentin, Snail and Slug as well as decreased expression of ZO-1 and E-cadherin compared with that of Lgr5-negative cells. In addition, we identified the co-expression of Lgr5 and the mesenchymal marker Vimentin through immunofluorescence staining. Furthermore, triple-positive cells migrated and invaded significantly more rapidly than their negative counterparts, which corresponds to previous results $[25,55]$. Collectively, our data suggest a partial overlap between the transcriptional programs underlying the EMT and Lgr5-positive cells.

CSCs are a subset of cells with indefinite passaging ability; they can continuously proliferate and differentiate [2, 29]. Therefore, CSCs have been identified as key factors in cancer growth and metastasis [56]. Accordingly, we assessed the proliferative capacity of each subpopulation using the CCK-8 assay. We found that Lgr5-positive cells had a significantly higher proliferative capacity than Lgr5-negative cells. More importantly, triple-positive cells had a significantly higher capacity to proliferate and differentiate than their negative counterparts (Fig. 4). These findings are further supported by the in vitro tumorigenicity assay and in vivo tumor formation study in mice. Using the colony formation assay and soft agar assay, we found that Lgr5-positive cells had a significantly higher colony formation capacity than Lgr5-negative cells and that the Lgr5+CD44+EpCAM+ subpopulation could generate more colonies than any other subpopulation, indicating a higher tumorigenic potential in vitro. Moreover, we found that tumors formed earlier and were significantly larger in mice transplanted with Lgr5+CD44+EpCAM+ cells than in mice transplanted with Lgr5+CD44+EpCAM- or Lgr5+CD44-EpCAM+ cells. In addition, no tumor growth was observed after injection of even $1 \times 10^{7}$ Lgr5-CD44+EpCAM+ or Lgr5-CD44EpCAM- cells (data not shown). The volumes of the tumors generated by $5 \times 10^{5}$ cells from each subpopulation are shown in Fig. 6.

For the first time, our systematic experimental data have shown that Lgr5 expression is highly restricted to colorectal CSCs. Most importantly, CSCs could be further identified and defined using Lgr5 combined with CD44 and EpCAM, which might open a new avenue for colorectal cancer cell eradication by targeting Lgr5+CD44+EpCAM+ cells.

\section{Acknowledgements}

This study was supported by the National Nature Science Foundation of China (Grants 81402444 and 81402357), the Key Project of Education Department of Sichuan Province (Grant 16ZA0226), the Chinese Ministry of Science and Technology (Grant 14C26213601925), the Health and Family Planning Commission of Chongqing City (Grant 2016MSXM021), and the Project of Science and Technology Department of Sichuan Province (Grant 2017JY0170). The funders had no role in study design, data collection and analysis, decision to publish, or preparation of the article.

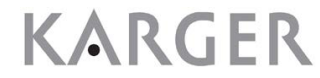




\section{Cellular Physiology Cell Physiol Biochem 2018;46:860-872 \begin{tabular}{ll|l} 
and Biochemistry Published online: April06, 2018 & $\begin{array}{l}\text { C) } 2018 \text { The Author(s). Published by S. Karger AG, Basel } \\
\text { www.karger.com/cpb }\end{array}$ \\
\hline
\end{tabular}}

Leng et al.: Lgr5+CD44+EpCAM+ Defines CSCs

\section{Disclosure Statement}

The authors declare no conflicts of interest.

\section{References}

1 Chen W, Zheng R, Baade PD, Zhang S, Zeng H, Bray F, Jemal A, Yu XQ He J: Cancer statistics in China, 2015. CA Cancer J Clin 2016;66:115-132.

-2 Zhou JY, Chen M, Ma L, Wang X, Chen YG, Liu SL: Role of CD44(high)/CD133(high) HCT-116 cells in the tumorigenesis of colon cancer. Oncotarget 2016;7:7657-7666.

-3 Ruscetti M, Quach B, Dadashian EL, Mulholland DJ, Wu H: Tracking and Functional Characterization of Epithelial-Mesenchymal Transition and Mesenchymal Tumor Cells during Prostate Cancer Metastasis. Cancer Res 2015;75:2749-2759.

4 Mani SA, Guo W, Liao MJ, Eaton EN, Ayyanan A, Zhou AY, Brooks M, Reinhard F, Zhang CC, Shipitsin M, Campbell LL, Polyak K, Brisken C, Yang J, Weinberg RA: The epithelial-mesenchymal transition generates cells with properties of stem cells. Cell 2008;133:704-715.

5 Bharti R, Dey G, Mandal M: Cancer development, chemoresistance, epithelial to mesenchymal transition and stem cells: A snapshot of IL-6 mediated involvement. Cancer Lett 2016;375:51-61.

6 Prieto-Garcia E, Diaz-Garcia CV, Garcia-Ruiz I, Agullo-Ortuno MT: Epithelial-to-mesenchymal transition in tumor progression. Med Oncol 2017;34:122.

7 Wang W, Lv Z, Yang Z, Zhou P, Dong J, Li F: Prrx1 enhanced the migration ability of breast cancer cell by initiating epithelial-mesenchymal transition. Cancer Cell Research 2017; 15:362-367.

8 Leng Z, Tao K, Xia Q, Tan J, Yue Z, Chen J, Xi H, Li J, Zheng H: Kruppel-like factor 4 acts as an oncogene in colon cancer stem cell-enriched spheroid cells. PLoS One 2013;8:e56082.

-9 Kemper K, Prasetyanti PR, De Lau W, Rodermond H, Clevers H, Medema JP: Monoclonal antibodies against Lgr5 identify human colorectal cancer stem cells. Stem Cells 2012;30:2378-2386.

10 Huang EH, Hynes MJ, Zhang T, Ginestier C, Dontu G, Appelman H, Fields JZ, Wicha MS, Boman BM: Aldehyde dehydrogenase 1 is a marker for normal and malignant human colonic stem cells (SC) and tracks SC overpopulation during colon tumorigenesis. Cancer Res 2009;69:3382-3389.

11 Jiang Y, Li W, He X, Zhang H, Jiang F, Chen Z: Lgr5 expression is a valuable prognostic factor for colorectal cancer: evidence from a meta-analysis. BMC Cancer 2016;16:12.

12 Srinivasan T, Walters J, Bu P, Than EB, Tung KL, Chen KY, Panarelli N, Milsom J, Augenlicht L, Lipkin SM, Shen X: NOTCH Signaling Regulates Asymmetric Cell Fate of Fast- and Slow-Cycling Colon Cancer-Initiating Cells. Cancer Res 2016;76:3411-3421.

13 Carmon KS, Lin Q, Gong X, Thomas A, Liu Q: LGR5 interacts and cointernalizes with Wnt receptors to modulate Wnt/beta-catenin signaling. Mol Cell Biol 2012;32:2054-2064.

-14 Vieira GC, Chockalingam S, Melegh Z, Greenhough A, Malik S, Szemes M, Park JH, Kaidi A, Zhou L, Catchpoole D, Morgan R, Bates DO, Gabb PD, Malik K: LGR5 regulates pro-survival MEK/ERK and proliferative Wnt/beta-catenin signalling in neuroblastoma. Oncotarget 2015;6:40053-40067.

-15 Yang L, Tang H, Kong Y, Xie X, Chen J, Song C, Liu X, Ye F, Li N, Wang N: LGR5 Promotes Breast Cancer Progression and Maintains Stem-Like Cells Through Activation of Wnt/beta-Catenin Signaling. Stem Cells 2015;33:2913-2924.

-16 Dalerba P, Dylla SJ, Park IK, Liu R, Wang X, Cho RW, Hoey T, Gurney A, Huang EH, Simeone DM, Shelton AA, Parmiani G, Castelli C, Clarke MF: Phenotypic characterization of human colorectal cancer stem cells. Proc Natl Acad Sci U S A 2007;104:10158-10163.

-17 Roy K, Kanwar RK, Kanwar JR: LNA aptamer based multi-modal, Fe304-saturated lactoferrin (Fe304bLf) nanocarriers for triple positive (EpCAM, CD133, CD44) colon tumor targeting and NIR, MRI and CT imaging. Biomaterials 2015;71:84-99.

18 Liu D, Sun J, Zhu J, Zhou H, Zhang X, Zhang Y: Expression and clinical significance of colorectal cancer stem cell marker EpCAMhigh/CD44+ in colorectal cancer. Oncol Lett 2014;7:1544-1548.

19 Hanahan D, Weinberg RA: Hallmarks of cancer: the next generation. Cell 2011;144:646-674.

20 Basu S, Haase G, Ben-Ze'ev A: Wnt signaling in cancer stem cells and colon cancer metastasis. F1000Res 2016;5 


\section{Cellular Physiology Cell Physiol Biochem 2018;46:860-872

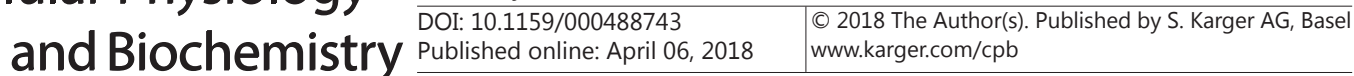

Leng et al.: Lgr5+CD44+EpCAM+ Defines CSCs

-21 Wang H, Liu B, Wang J, Li J, Gong Y, Li S, Wang C, Cui B, Xue X, Yang M, Fan W, Kang Z, Kamran M, Xu J, Tian P, Luo Y, Hou Z, Dong L, Ren Y, Li M, Wen Q, Cheng W, Xu L, Wang L, Liu Q: Reduction of NANOG Mediates the Inhibitory Effect of Aspirin on Tumor Growth and Stemness in Colorectal Cancer. Cell Physiol Biochem 2017;44:1051-1063.

22 Visvader JE, Lindeman GJ: Cancer stem cells in solid tumours: accumulating evidence and unresolved questions. Nat Rev Cancer 2008;8:755-768.

-23 Nolte SM, Venugopal C, McFarlane N, Morozova O, Hallett RM, O’Farrell E, Manoranjan B, Murty NK, Klurfan P, Kachur E, Provias JP, Farrokhyar F, Hassell JA, Marra M, Singh SK: A cancer stem cell model for studying brain metastases from primary lung cancer. J Natl Cancer Inst 2013;105:551-562.

-24 Reynolds BA, Weiss S: Clonal and population analyses demonstrate that an EGF-responsive mammalian embryonic CNS precursor is a stem cell. Dev Biol 1996;175:1-13.

25 Ren D, Villeneuve NF, Jiang T, Wu T, Lau A, Toppin HA, Zhang DD: Brusatol enhances the efficacy of chemotherapy by inhibiting the Nrf2-mediated defense mechanism. Proc Natl Acad Sci U S A 2011;108:1433-1438.

26 Chen T, Liu Y, Lin B, He R, Huang Z: Effects of RNA interference of the eno1 gene on the malignant biological behaviors of gastric cell lines. Cancer Cell Research 2017; 14: 326-333.

27 Wei D, Kanai M, Huang S, Xie K: Emerging role of KLF4 in human gastrointestinal cancer. Carcinogenesis 2006;27:23-31.

28 Kokolus KM, Capitano ML, Lee CT, Eng JW, Waight JD, Hylander BL, Sexton S, Hong CC, Gordon CJ, Abrams SI, Repasky EA: Baseline tumor growth and immune control in laboratory mice are significantly influenced by subthermoneutral housing temperature. Proc Natl Acad Sci U S A 2013;110:20176-20181.

-29 Du L, Li YJ, Fakih M, Wiatrek RL, Duldulao M, Chen Z, Chu P, Garcia-Aguilar J, Chen Y: Role of SUMO activating enzyme in cancer stem cell maintenance and self-renewal. Nat Commun 2016;7:12326.

-30 Tropepe V, Sibilia M, Ciruna BG, Rossant J, Wagner EF, van der Kooy D: Distinct neural stem cells proliferate in response to EGF and FGF in the developing mouse telencephalon. Dev Biol 1999;208:166-188.

-31 Yin T, Wei H, Leng Z, Yang Z, Gou S, Wu H, Zhao G, Hu X, Wang C: Bmi-1 promotes the chemoresistance, invasion and tumorigenesis of pancreatic cancer cells. Chemotherapy 2011;57:488-496.

-32 Muraro MG, Mele V, Daster S, Han J, Heberer M, Cesare Spagnoli G, Iezzi G: CD133+, CD166+CD44+, and CD24+CD44+ phenotypes fail to reliably identify cell populations with cancer stem cell functional features in established human colorectal cancer cell lines. Stem Cells Transl Med 2012;1:592-603.

33 Xu L, Chi C, Wang C, Zhang L: Association between bevacizumab-related chemotherapy regimens and serum vascular endothelial growth factor-A level in patients with metastatic colorectal cancer. Cancer Cell Research 2015; 5: 120-124

34 Li J, Zhang N, Zhang R, Sun L, Yu W, Guo W, Gao Y, Li M, Liu W, Liang P, Deng W, Cui X: CDC5L Promotes hTERT Expression and Colorectal Tumor Growth. Cell Physiol Biochem 2017;41:2475-2488.

-35 Zhang N, Xie Y, Tai Y, Gao Y, Guo W, Yu W, Li J, Feng X, Hao J, Gao Y, Zhao X, Liao Y, Jiang W, Liu G, Deng W, Cui X: Bufalin Inhibits hTERT Expression and Colorectal Cancer Cell Growth by Targeting CPSF4. Cell Physiol Biochem 2016;40:1559-1569.

-36 Rios AC, Fu NY, Lindeman GJ, Visvader JE: In situ identification of bipotent stem cells in the mammary gland. Nature 2014;506:322-327.

-37 Barker N, van Es JH, Kuipers J, Kujala P, van den Born M, Cozijnsen M, Haegebarth A, Korving J, Begthel H, Peters PJ, Clevers H: Identification of stem cells in small intestine and colon by marker gene Lgr5. Nature 2007;449:1003-1007.

-38 Wang C, Yue Y, Shao B, Qiu Z, Mu J, Tang J, Han X, Xiang T, Ren G: Dickkopf-Related Protein 2 is Epigenetically Inactivated and Suppresses Colorectal Cancer Growth and Tumor Metastasis by Antagonizing Wnt/beta-Catenin Signaling. Cell Physiol Biochem 2017;41:1709-1724.

39 Yu W, Li L, Zheng F, Yang W, Zhao S, Tian C, Yin W, Chen Y, Guo W, Zou L, Deng W: beta-Catenin Cooperates with CREB Binding Protein to Promote the Growth of Tumor Cells. Cell Physiol Biochem 2017;44:467-478.

40 Yamashita T, Budhu A, Forgues M, Wang XW: Activation of hepatic stem cell marker EpCAM by Wnt-betacatenin signaling in hepatocellular carcinoma. Cancer Res 2007;67:10831-10839.

41 Lugli A, Iezzi G, Hostettler I, Muraro MG, Mele V, Tornillo L, Carafa V, Spagnoli G, Terracciano L, Zlobec I: Prognostic impact of the expression of putative cancer stem cell markers CD133, CD166, CD44s, EpCAM, and ALDH1 in colorectal cancer. Br J Cancer 2010;103:382-390. 


\section{Cellular Physiology Cell Physiol Biochem 2018;46:860-872 \begin{tabular}{l|l} 
DOI: 10.1159/000488743 & $\begin{array}{l}\text { O 2018 The Author(s). Published by S. Karger AG, Basel } \\
\text { www.karger.com/cpb }\end{array}$ \\
\hline
\end{tabular} \\ Leng et al.: Lgr5+CD44+EpCAM+ Defines CSCs}

42 Piscuoglio S, Lehmann FS, Zlobec I, Tornillo L, Dietmaier W, Hartmann A, Wunsch PH, Sessa F, Rummele P, Baumhoer D, Terracciano LM: Effect of EpCAM, CD44, CD133 and CD166 expression on patient survival in tumours of the ampulla of Vater. J Clin Pathol 2012;65:140-145.

43 Belov L, Zhou J, Christopherson RI: Cell surface markers in colorectal cancer prognosis. Int J Mol Sci 2010;12:78-113.

44 Kuhn S, Koch M, Nubel T, Ladwein M, Antolovic D, Klingbeil P, Hildebrand D, Moldenhauer G, Langbein L, Franke WW, Weitz J, Zoller M: A complex of EpCAM, claudin-7, CD44 variant isoforms, and tetraspanins promotes colorectal cancer progression. Mol Cancer Res 2007;5:553-567.

-45 O’Brien CA, Pollett A, Gallinger S, Dick JE: A human colon cancer cell capable of initiating tumour growth in immunodeficient mice. Nature 2007;445:106-110.

46 Younesi V, Shirazi FG, Memarian A, Amanzadeh A, Jeddi-Tehrani M, Shokri F: Assessment of the effect of TLR7/8, TLR9 agonists and CD40 ligand on the transformation efficiency of Epstein-Barr virus in human B lymphocytes by limiting dilution assay. Cytotechnology 2014;66:95-105.

47 Takahashi K, Yamanaka S: Induction of pluripotent stem cells from mouse embryonic and adult fibroblast cultures by defined factors. Cell 2006;126:663-676.

48 Khorrami S, Zavaran Hosseini A, Mowla SJ, Malekzadeh R: Verification of ALDH Activity as a Biomarker in Colon Cancer Stem Cells-Derived HT-29 Cell Line. Iran J Cancer Prev 2015;8:e3446.

49 Saigusa S, Tanaka K, Toiyama Y, Yokoe T, Okugawa Y, Ioue Y, Miki C, Kusunoki M: Correlation of CD133, OCT4, and SOX2 in rectal cancer and their association with distant recurrence after chemoradiotherapy. Ann Surg Oncol 2009;16:3488-3498.

50 Saiki Y, Ishimaru S, Mimori K, Takatsuno Y, Nagahara M, Ishii H, Yamada K, Mori M: Comprehensive analysis of the clinical significance of inducing pluripotent stemness-related gene expression in colorectal cancer cells. Ann Surg Oncol 2009;16:2638-2644.

51 Gao D, Vahdat LT, Wong S, Chang JC, Mittal V: Microenvironmental regulation of epithelial-mesenchymal transitions in cancer. Cancer Res 2012;72:4883-4889.

-52 Yin X, Zhang BH, Zheng SS, Gao DM, Qiu SJ, Wu WZ, Ren ZG: Coexpression of gene Oct4 and Nanog initiates stem cell characteristics in hepatocellular carcinoma and promotes epithelial-mesenchymal transition through activation of Stat3/Snail signaling. J Hematol Oncol 2015;8:23.

-53 Yang Y, Wang G, Zhu D, Huang Y, Luo Y, Su P, Chen X, Wang Q: Epithelial-mesenchymal transition and cancer stem cell-like phenotype induced by Twist1 contribute to acquired resistance to irinotecan in colon cancer. Int J Oncol 2017;51:515-524.

54 Choi JE, Bae JS, Kang MJ, Chung MJ, Jang KY, Park HS, Moon WS: Expression of epithelial-mesenchymal transition and cancer stem cell markers in colorectal adenocarcinoma: Clinicopathological significance. Oncol Rep 2017

55 Eisenberg MC, Kim Y, Li R, Ackerman WE, Kniss DA, Friedman A: Mechanistic modeling of the effects of myoferlin on tumor cell invasion. Proc Natl Acad Sci U S A 2011;108:20078-20083.

-56 Ordonez-Moran P, Dafflon C, Imajo M, Nishida E, Huelsken J: HOXA5 Counteracts Stem Cell Traits by Inhibiting Wnt Signaling in Colorectal Cancer. Cancer Cell 2015;28:815-829. 